\title{
Expression and functional analysis of the rice plasma-membrane intrinsic protein gene family
}

\author{
Lei Guo ${ }^{1}$, Zi Yi Wang ${ }^{1}$, Hong Lin ${ }^{1}$, Wei Er Cui ${ }^{1}$, Jun Chen ${ }^{1}$, Meihua Liu ${ }^{1}$, Zhang Liang Chen ${ }^{1,2}$, Li Jia Qu ${ }^{1,2}$, \\ Hongya $\mathrm{Gu}^{1,2}$
}

${ }^{1}$ Peking-Yale Joint Research Center for Plant Molecular Genetics and AgroBiotechnology, National Laboratory for Protein Engineering and Plant Genetic Engineering, College of Life Sciences, Peking University, Beijing 100871, China; ${ }^{2}$ The National Plant Gene Research Center, Beijing 100101, China

Plasma membrane intrinsic proteins (PIPs) are a subfamily of aquaporins that enable fast and controlled translocation of water across the membrane. In this study, we systematically identified and cloned ten PIP genes from rice. Based on the similarity of the amino acid sequences they encoded, these rice $P I P$ genes were classified into two groups and designated as $O S P I P 1-1$ to $O S P I P 1-3$ and $O S P I P 2-1$ to OsPIP2-7 following the nomenclature of PIP genes in maize. Quantitative RT-PCR analysis identified three root-specific and one leaf-specific $O S P I P$ genes. Furthermore, the expression profile of each OSPIP gene in response to salt, drought and ABA treatment was examined in detail. Analysis on transgenic plants over-expressing of either OsPIP1 (OsPIP1-1) or OsPIP2 (OsPIP2-2) in wild-type Arabidopsis, showed enhanced tolerance to salt $(100 \mathrm{mM}$ of $\mathrm{NaCl})$ and drought $(200 \mathrm{mM}$ of mannitol), but not to salt treatment of higher concentration (150 $\mathrm{mM}$ of $\mathrm{NaCl}$ ). Taken together, these data suggest a distinct role of each OsPIP gene in response to different stresses, and should add a new layer to the understanding of the physiological function of rice PIP genes.

Cell Research (2006) 16:277-286. doi:10.1038/sj.cr.7310035; published online 16 March 2006

Keywords: aquaporins, PIP, quantitative RT-PCR, Oryza sativa, organ-specific

\section{Introduction}

Water is a fundamental, yet the most essential solvent in all organisms. Transmembrane water transport occurs by diffusion through the lipid bilayer and/or by increased membrane permeability due to the water channel proteins, aquaporins [1]. The presence of aquaporins, a class of major intrinsic proteins (MIPs), enables fast and controlled translocation of water across the membrane. Aquaporins have been found in bacteria, fungi, animals and plants. Plant aquaporins are classified into four major groups: the tonoplast intrinsic proteins (TIPs) localized to the vacuolar membranes, the plasma membrane intrinsic proteins (PIPs)

Correspondence: Hong Ya Gu

Tel: 86-10-6275-1847; Fax: 86-10-6275-1841;

E-mail: guhy@pku.edu.cn

Received 4 Jul 2005; revised 30 Sep 2005; Accepted 17 Oct 2005, published online 16 March 2006 localized to the plasma membranes, the NOD-26-like MIPs (NIPs), and the recently characterized small basic intrinsic proteins (SIPs) [2-5].

The structures of aquaporins have been intensively studied, revealing the functions of their conserved motifs and post-translational modification sites, in water transport [3-15]. The expression patterns of aquaporin genes in response to salt, drought, cold, submergence, light, and phytohormones have also been characterized in various plants [16-24]. Some aquaporins are tissue-specific, such as $\gamma$-MIP in shoots of rice [21], $\gamma$-TIP in seeds of Brassica napus [16] and ZmTIP2-3 in roots of maize [25].

Aquaporins have been systematically identified and classified in Arabidopsis and maize. There were thirteen PIP genes among the identified thirty and thirty-one aquaporin genes in Arabidopsis and maize, respectively [13, 26]. The function and expression patterns of the PIP gene family have been intensively studied in Arabidopsis, Brassicca, and maize, especially in response to abiotic stress [7, 17, 
$18,26-29]$.

However, little is known about the aquaporin gene family in rice. Up to date, three PIP genes have been identified in rice, one of which was shown to have water transport activity in the Xenopus oocytes [30, 31]. The completion of rice genome sequence enables us to systematically identify the rice aquaporin genes and analyze their structure, biochemical property and physiological function.

In this paper, we identified and structurally analyzed 10 rice $P I P$ genes $(O s P I P s)$ that were classified into two subgroups (OSPIP1 and OsPIP2). Utilizing the quantitative RT-PCR method, we analyzed the response to abiotic stress by exploring their expression profile under various treatments, including salinity, drought, and ABA. Moreover, to further investigate the function of rice PIP proteins, we examined the tolerance of transgenic Arabidopsis plants over-expressing the rice PIP genes to salinity and drought.

\section{Materials and Methods}

\section{Plant material and stress treatment}

A rice cultivar (Oryza sativa L. spp. japonica cv. Zhonghua 11) and Arabidopsis thaliana (Columbia ecotype) were used in this study. Rice seeds were surface-sterilized and germinated on N6 solid medium containing 3\% sucrose, pH5.8. Rice seedlings of 3-d-old were transplanted into N6 liquid medium containing 3\% sucrose, and grow at $25-28^{\circ} \mathrm{C}$ under continuous light.

For salinity, drought and ABA treatments, 2-week-old rice seedlings were placed in the fresh N6 liquid medium containing $250 \mathrm{mM}$ of $\mathrm{NaCl}$ (salinity stress), 10\% PEG6000 (drought stress) and $50 \mu \mathrm{M}$ of ABA $[16,31,32]$, respectively. During the treatment, leaves and roots were collected separately at different time points and immediately frozen in liquid nitrogen. Eight individual seedlings were used for each time point and treatment, and the experiments were independently repeated three times.

\section{RNA isolation and cDNA synthesis}

Total RNAs of the leaves and roots of rice seedlings were isolated using the Plant RNeasy extraction kit (Qiagen, USA) and then digested with RNase-free DNaseI (Boeheringer Mannheim, Germany) according to the manufacture's instruction. RNA concentration was measured by spectrophotometer and RNA quality was examined in $1.2 \%$ formaldehyde agarose gel. Five microgram of total RNA was used to synthesize cDNA using SuperScript ${ }^{\mathrm{TM}}$ II RNase $\mathrm{H}^{-}$Reverse Transcriptase Kit (Invitrogen, USA) according to the manufacture's instruction.

\section{Primer design and cDNA cloning}

The sequences of primers for PCR amplification of full-length cDNAs of OsPIP genes and for quantitative RT-PCR are listed on Supplement 1. DNA sequence comparisons were made to ensure that each pair of primers is specific to the corresponding OSPIP genes. Amplified cDNAs were cloned into T-vector (Promega, USA) and sequenced.

Quantitative RT-PCR and quantification of transcript copy numbers of OsPIP genes

The quantitative RT-PCR was carried out in the DNA Engine OPTICON 2 Continuous Fluorescence Detector (MJ research, USA), using DyNAmo SYBR Green qPCR kit (Finnzymmes, Finland). PCR amplifications were performed in a total volume of $20 \mu \mathrm{l}$, containing $2 \mu 1$ cDNA (1:10 diluted, corresponding to about 40 ng RNA), 100 $\mathrm{nM}$ of each gene specific primer and $10 \mu 12 \times$ SYBR (Finnzymmes, Finland). Each PCR reaction was performed in three or four parallel independent experiments, using the following conditions: $95^{\circ} \mathrm{C}$ $5 \mathrm{~min} ; 40$ cycles of $15 \mathrm{~s}$ at $94^{\circ} \mathrm{C}, 30 \mathrm{~s}$ at $55^{\circ} \mathrm{C}, 12 \mathrm{~s}$ at $72^{\circ} \mathrm{C}$, and plate read. The data collection was carried out during the extension step. For a negative control, the PCR was performed without cDNA templates. The absolute and relative transcript copy number of OsPIP genes in each treated sample was calculated following the method described by Jang et al. [17], except that a rice actin gene $(O S A C T 1)$ was used to calculate the correction factor instead of the Arabidopsis actin gene.

\section{Plant transformation and root elongation analysis}

OsPIP1-1 and OSPIP2-2 cDNAs were cloned into pQGIE110, and transformed into the Agrobacterium GV3101. The cDNAs were then transformed into the wild-type Arabidopsis by floral dip method [33]. The total RNAs were extracted from transgenic seedlings as described above. The over-expression of OSPIP1-1 and OSPIP2-2 in transgenic plants was confirmed by RT-PCR.

Seeds of transgenic plants with OsPIPI-1 (line 7 and line 10), OSPIP2-2 (line 8 and line 19), and pQGIE110 vector were surfacesterilized and sown onto Murashige and Skoog (MS) solid medium [34] at $4{ }^{\circ} \mathrm{C}$ for vernalization. After three days, the plates were placed vertically under normal growth condition in a growth chamber (Percival, USA). About $4-5 \mathrm{~d}$ later the healthy seedlings were transferred to fresh MS medium with or without $100 \mathrm{mM}$ of $\mathrm{NaCl}, 150 \mathrm{mM}$ of $\mathrm{NaCl}$, and $200 \mathrm{mM}$ of mannitol. The plates were then placed vertically in the growth chamber again, but with the seedlings up-side-down [35]. The root length of each seedling was measured everyday for 7 $\mathrm{d}$ and analyzed by Image $\mathrm{J}$ software (NIH, USA).

\section{Results}

\section{Identification and sequence analysis of OsPIPs in rice}

After a systematic search over the NCBI database, rice genome sequence databases of the Beijing Genomics Institute (http://rise.genomics.org.cn), and the International Genome Research Program Website in Japan (http://rgp. dna.affrc.go.jp) using the cDNA sequences of 13 maize $P I P$ genes as queries, we identified 10 rice genes that encode the plasma membrane intrinsic proteins (OSPIPs). The cDNAs (coding region and 3'-UTR) of the 10 rice PIP genes were amplified by RT-PCR, cloned and sequenced. The 10 genes were divided into two groups based on the similarity of the amino acid sequence they encoded and the phylogenetic analysis (Figure 1A and 1B), and were designated as OsPIP1-1 (OsPIP1a), OsPIP1-2, OsPIP1-3 (RWC-3), OsPIP2-1 (OsPIP2a), OsPIP2-2, OsPIP2-3, OsPIP2-4, OsPIP2-5, OsPIP2-6, and OsPIP2-7 following the nomenclature of $P I P$ genes in maize $[31,32]$.

The DNA sequences of six OsPIP genes (i.e. OsPIP1-1, 
A
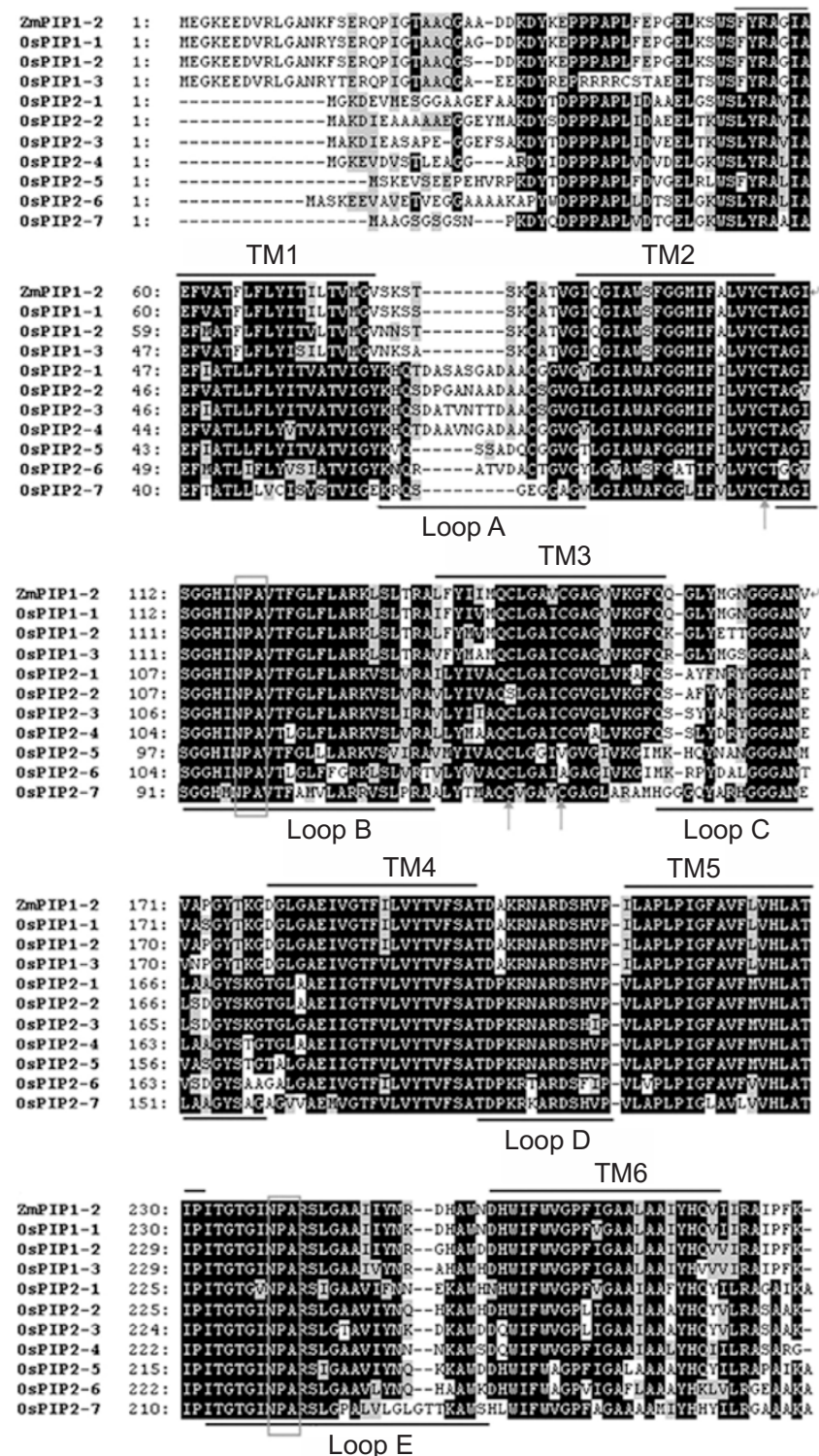

ZMPIP1-2 287: --SRS-----

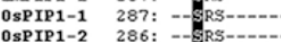

OSPIP1-3 288: -

OSPIP2-1 283: LGSFRSIX--

OsPIP2-2 282: LGSSSSFRG--

0SPIP2-3 281: LGSYRSTIA--

OSPIP2-4 279: VGFPRSIR--

OSPIP2-6 280: LGPRSNPSN-

$\begin{array}{ll}\text { 0SPIP2-6 } & 280: \text { LSFPRSTSVTA } \\ \text { 0SPIP2-7 } & 270: \text { FASSSYRSPHF }\end{array}$
$B$

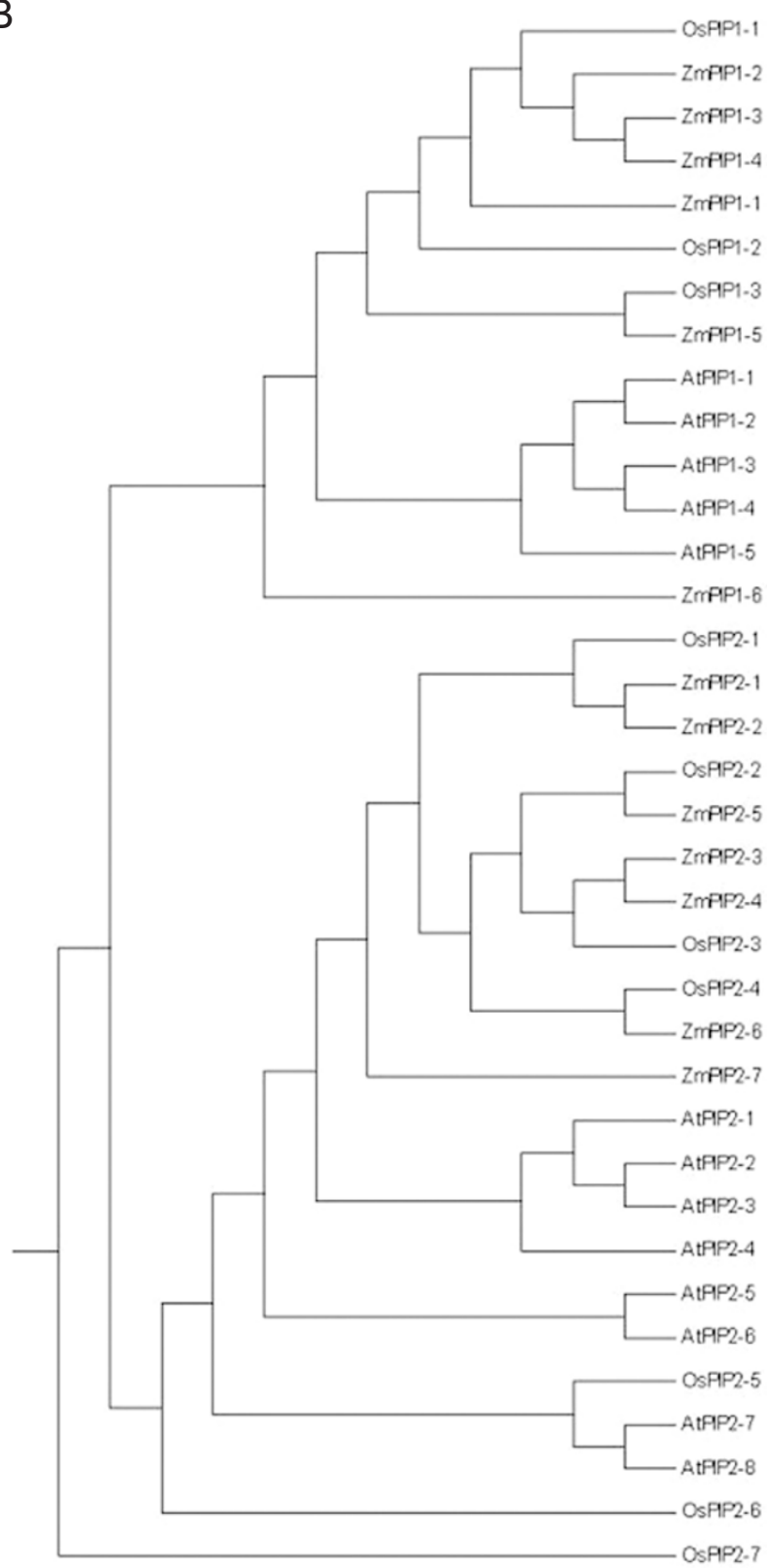

Figure 1 Deduced amino acid sequences of OsPIP proteins and their phylogenetic relationships. (A) Alignment and the deduced structure of the OsPIP proteins. Two conserved NPA motifs in loop B and loop E, and putative phosphorylation sites of PIP2 at C-terminus are boxed. Putative mercury sensitive sites are indicated by arrows. Putative bilayer-spanning domains (TM 1-6) and the connecting loops (A-E) are indicated by solid line. (B) Phylogenetic tree of PIP proteins from rice (OsPIP), maize (ZmPIP) and Arabidopsis (AtPIP) based on the deduced amino acid sequences aligned by Clustal W.

OsPIP1-2, OsPIP2-1, OsPIP2-2, OsPIP2-3, and OsPIP27) were identical to those in the databases, while the sequences of OsPIP1-3, OsPIP2-4, OsPIP2-5, and OsPIP2-6 were slightly different from those in the databases. The differences in DNA sequences did not alter their encoded amino acid sequences. The OsPIP proteins contained 280 to 
A

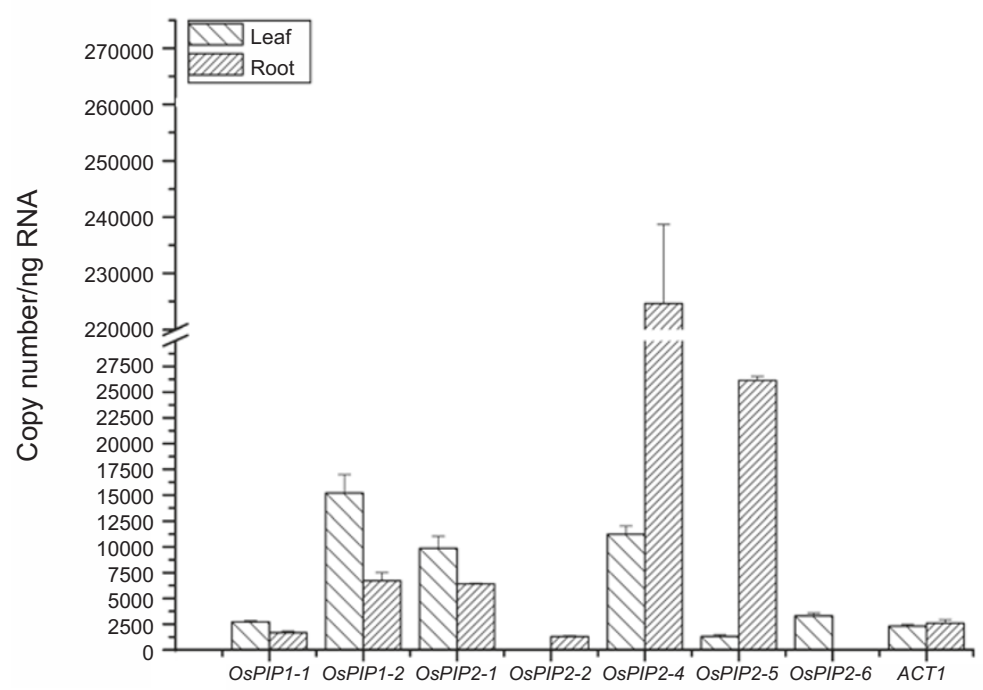

B

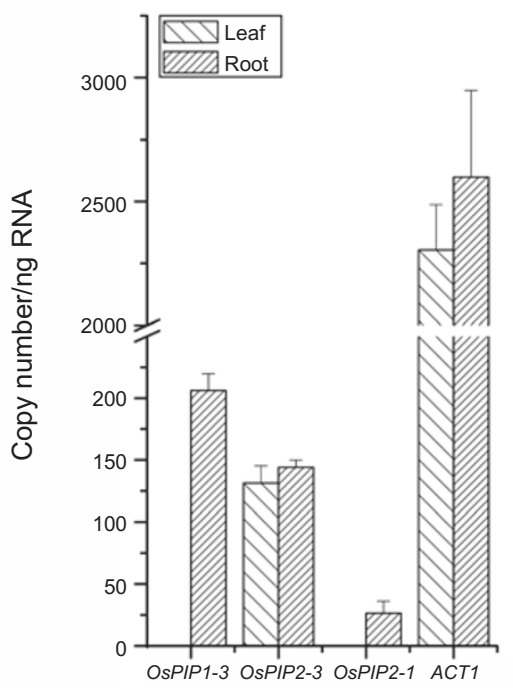

Figure 2 Absolute transcript copy numbers of each OsPIP gene in the leaves and roots of 2-week-old rice seedlings. (A) The genes with transcript copy numbers more than $10^{3}$. (B) The genes with transcript copy numbers less than 210 . Error bars: standard errors.
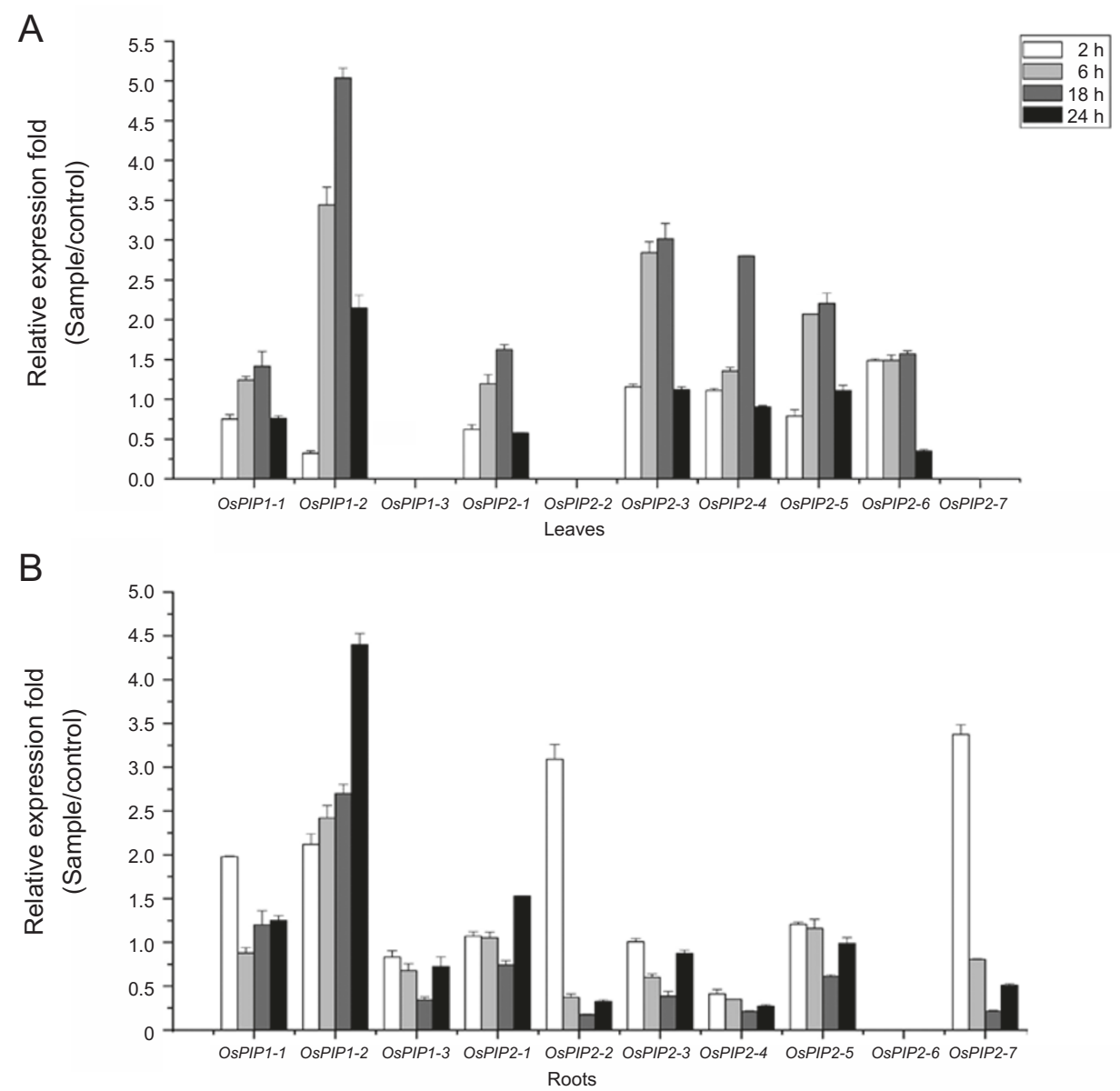

Figure 3 Relative expression levels of the $O S P I P$ genes in response to ABA in the leaves and roots of rice seedlings. (A) Relative expression level of the OSPIP genes in the leaves. (B) Relative expression level of the OsPIP genes in the roots. The transcript levels of each $O S P I P$ gene in the leaves and roots of treated seedlings were plotted as the relative expression fold of the non-treated seedlings $(0 \mathrm{~h})$ for 2, 6, 18 and $24 \mathrm{~h}$. Error Bars: standard errors. 
A

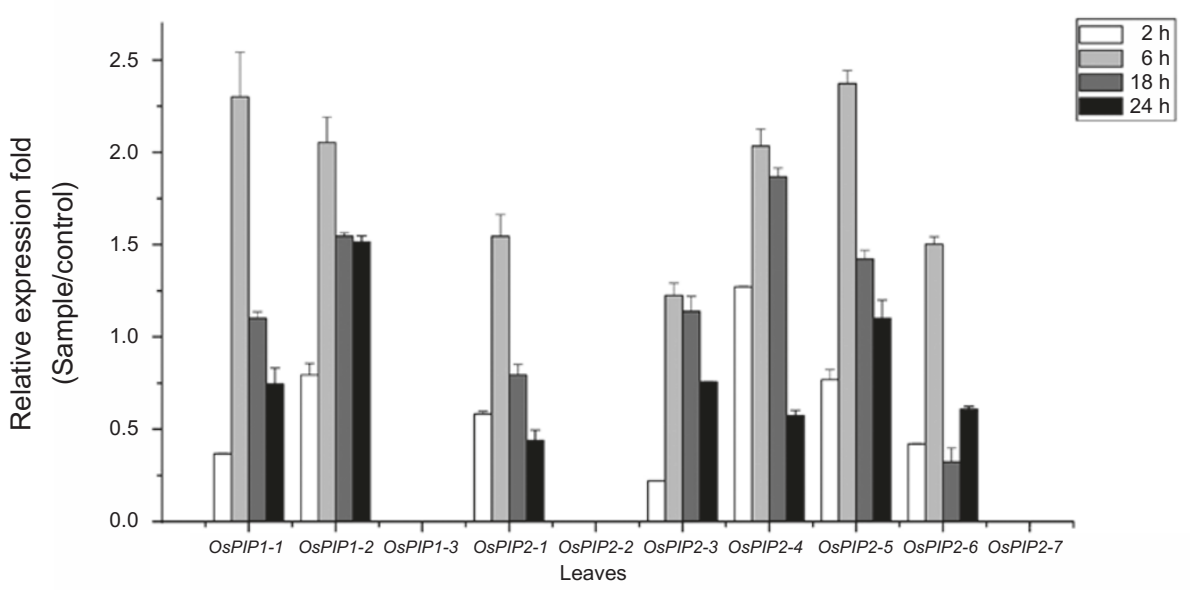

B

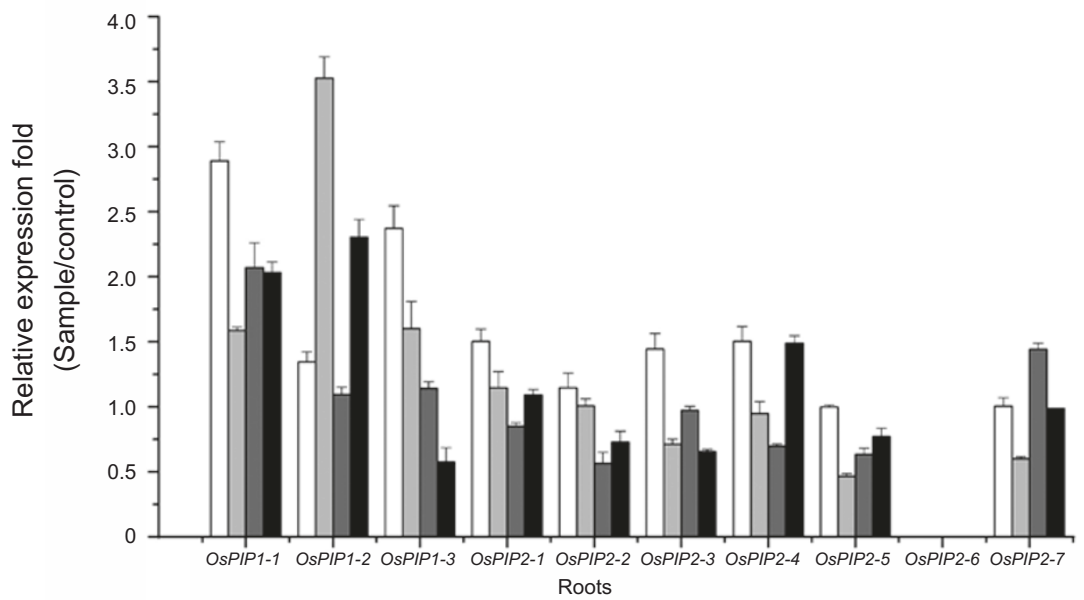

Figure 4 Relative expression levels of the $O S P I P$ genes in response to $\mathrm{NaCl}$ in the leaves and roots of rice seedlings. (A) Relative expression level of the OSPIP genes in the leaves. (B) Relative expression level of the OsPIP genes in the roots. The transcript levels of each $O S P I P$ gene in the leaves and roots of treated seedlings were plotted as the relative expression fold of the non-treated seedlings for 2, 6, 18 and $24 \mathrm{~h}$. Error Bars: standard errors.

290 amino acid residues, and the sequence similarity among them ranged from 57\% (between OsPIP1-3 and OsPIP2-7) to $92 \%$ (between OsPIP1-1 and OsPIP1-2). The 10 OsPIP proteins were found sharing six conserved transmembrane domains (TMs) and five connecting loops (Figure 1A) [30, 36, 37]. Moreover, the sequences around the NPA motifs (Asn-Pro-Ala) were highly conserved (Figure 1A).

Expression profile of the 10 OsPIP genes in rice seedlings

To further characterize the function of these $O S P I P$ genes, we examined the transcript level of each OsPIP gene using quantitative RT-PCR method in the leaves and roots of 2-week-old rice seedlings. The results showed that all the OSPIP genes had transcript copy numbers smaller than $2.6 \times 10^{4}$ per nanogram of total RNA, except for OsPIP2-4 whose copy number in root was ten folds larger $\left(2.2 \times 10^{5}\right)$. Based on the copy numbers, the rice OSPIP genes could roughly be divided into two groups, one with the copy number larger than $10^{3}$ (Figure $2 \mathrm{~A}$ ) and the other with the copy number smaller than 210 (Figure 2B). OsPIP27 had the lowest transcript level in roots, only 26 copies per nanogram of total RNA (Figure 2B). As an internal control, the transcript of the rice OsACT1 gene in roots and leaves was between $2000-3000$ copies per nanogram of total RNA.

Interestingly, the result also showed that the expression of three genes, OsPIP1-3, OsPIP2-2 and OsPIP2-7, could be detected only in roots, whereas that of OSPIP2-6 was found leaf-specific (Figure 2A and 2B), at this stage of growth. The expression of the other six genes were detected, although differentially, both in leaves and roots. 
The transcript levels of OSPIP1-1, OsPIP1-2 and OSPIP2-1 were 1.5-2.3 times higher in leaves than in roots, whereas the expression levels of OSPIP2-4 and OSPIP2-5 were found 20 fold higher in roots than in leaves. OsPIP2-3 was the only member whose expression was found no significant difference between the two organs (Figure 2), at this stage.

The expression of the OsPIP genes in response to $A B A$

In order to investigate the role of OSPIP genes in stress response, we examined the expression levels of the $O s P I P$ genes in rice leaves and roots when treated with $50 \mu \mathrm{M}$ of ABA at different time intervals, by quantitative RT-PCR. In response to this stimulus, all of the OSPIP genes shared a similar expression time course pattern in rice leaves, that is, the transcript level, although varied at $2 \mathrm{~h}$, increased at 6 $\mathrm{h}$, peaked at $18 \mathrm{~h}$, but decreased at $24 \mathrm{~h}$ (Figure $3 \mathrm{~A}$ ).

The expression pattern of OsPIPs in roots varied significantly from that in leaves. As shown in Figure 3B, the expression of most OsPIP genes were not much altered or rather slightly depressed during $24 \mathrm{~h} \mathrm{ABA}$ treatment. However, both $O S P I P 2-2$ and $O S P I P 2-7$ were immediately induced to 3-3.5 fold of the control at $2 \mathrm{~h}$ of the treatment, and then rapidly decreased to half of the control at $6 \mathrm{~h}$ (Figure 3B). Meanwhile, the expression of OsPIP1-3, OsPIP2-3 and $O S P I P 2-4$ were much suppressed under the treatment at all indicated time points (Figure 3B).

The expression of the OsPIP genes in response to salt and drought
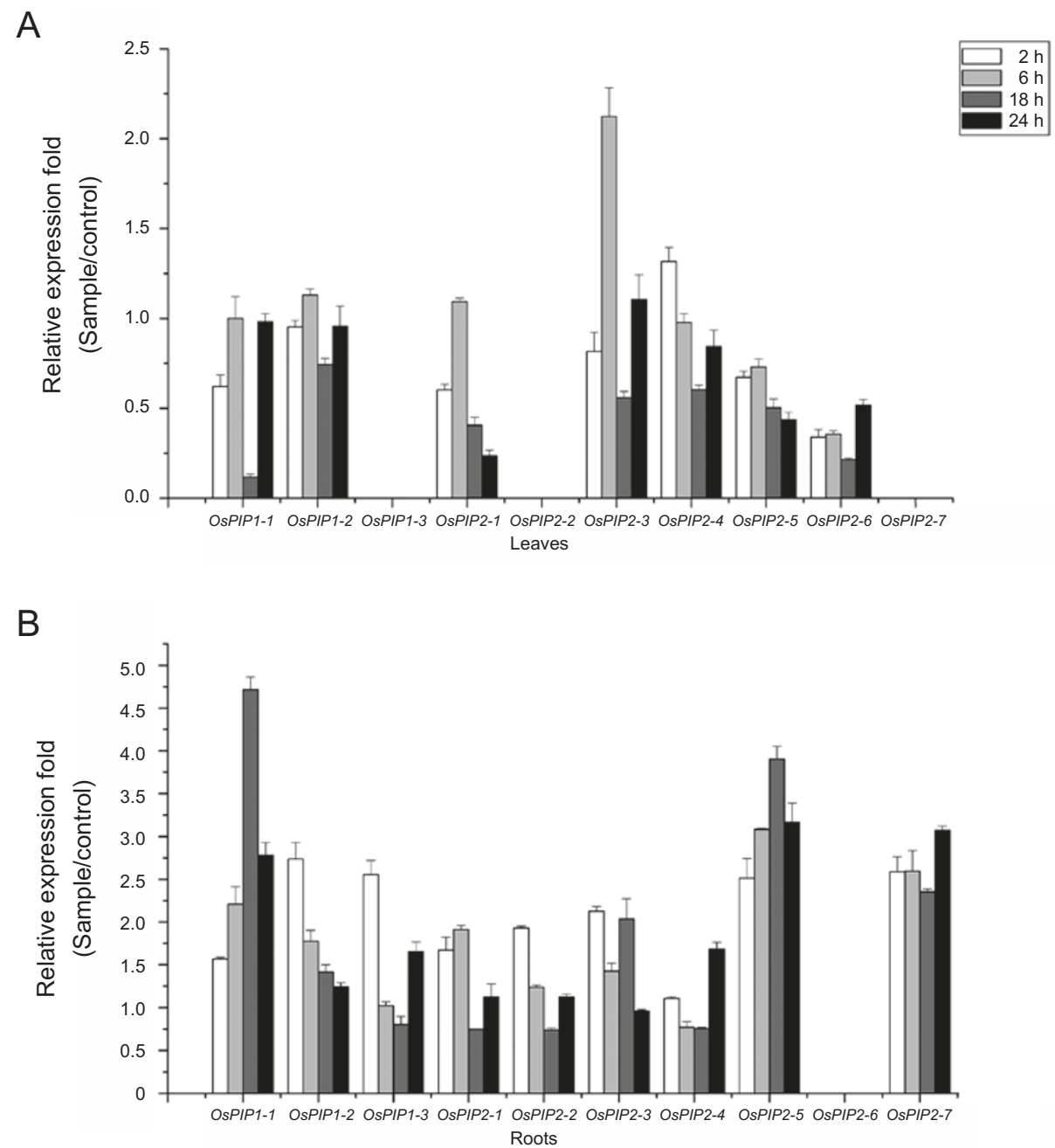

Figure 5 Relative expression levels of the 10 OsPIP genes in response to PEG6000 in the leaves and roots of rice seedlings. (A) Relative expression level of the 10 OSPIP genes in the leaves. (B) Relative expression level of the 10 OsPIP genes in the roots. The transcript levels of each $O_{S} P I P$ in the leaves and roots of treated seedlings were plotted as the relative expression fold of the nontreated seedlings for 2, 6, 18 and $24 \mathrm{~h}$. Error Bars: standard errors. 
A
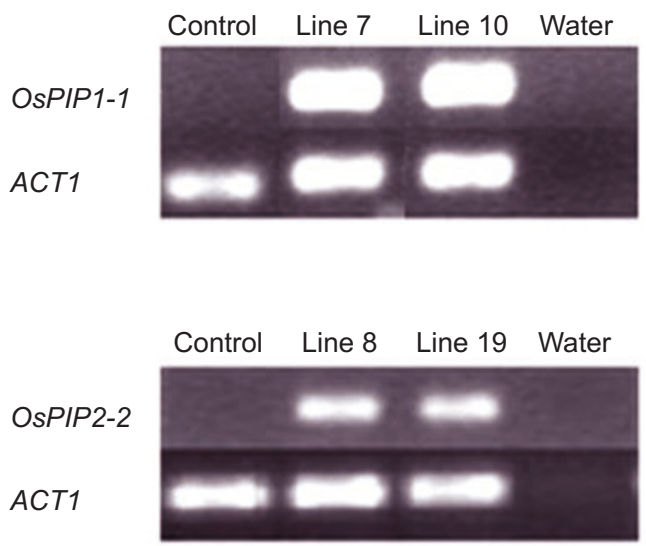

C
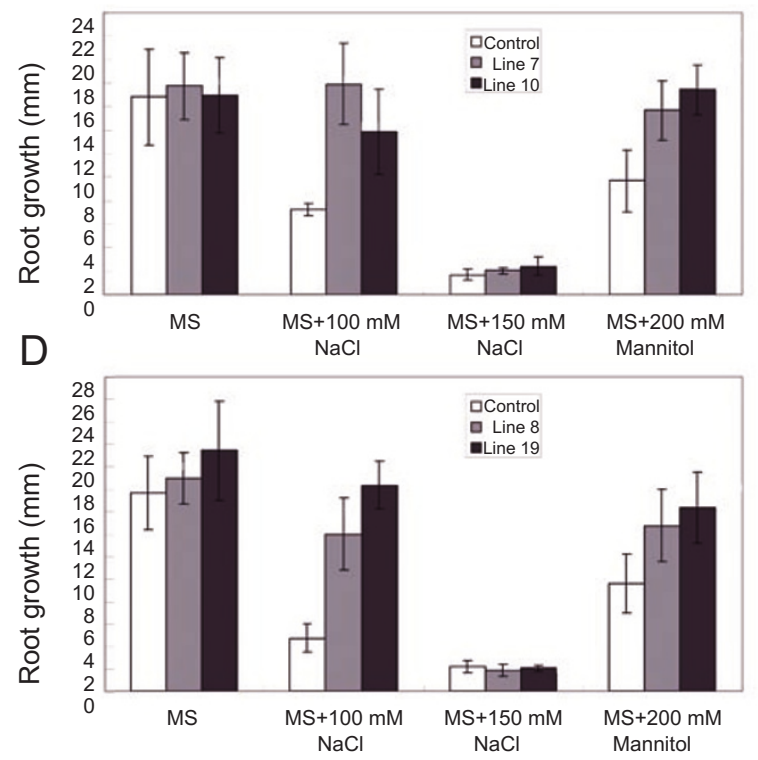

B
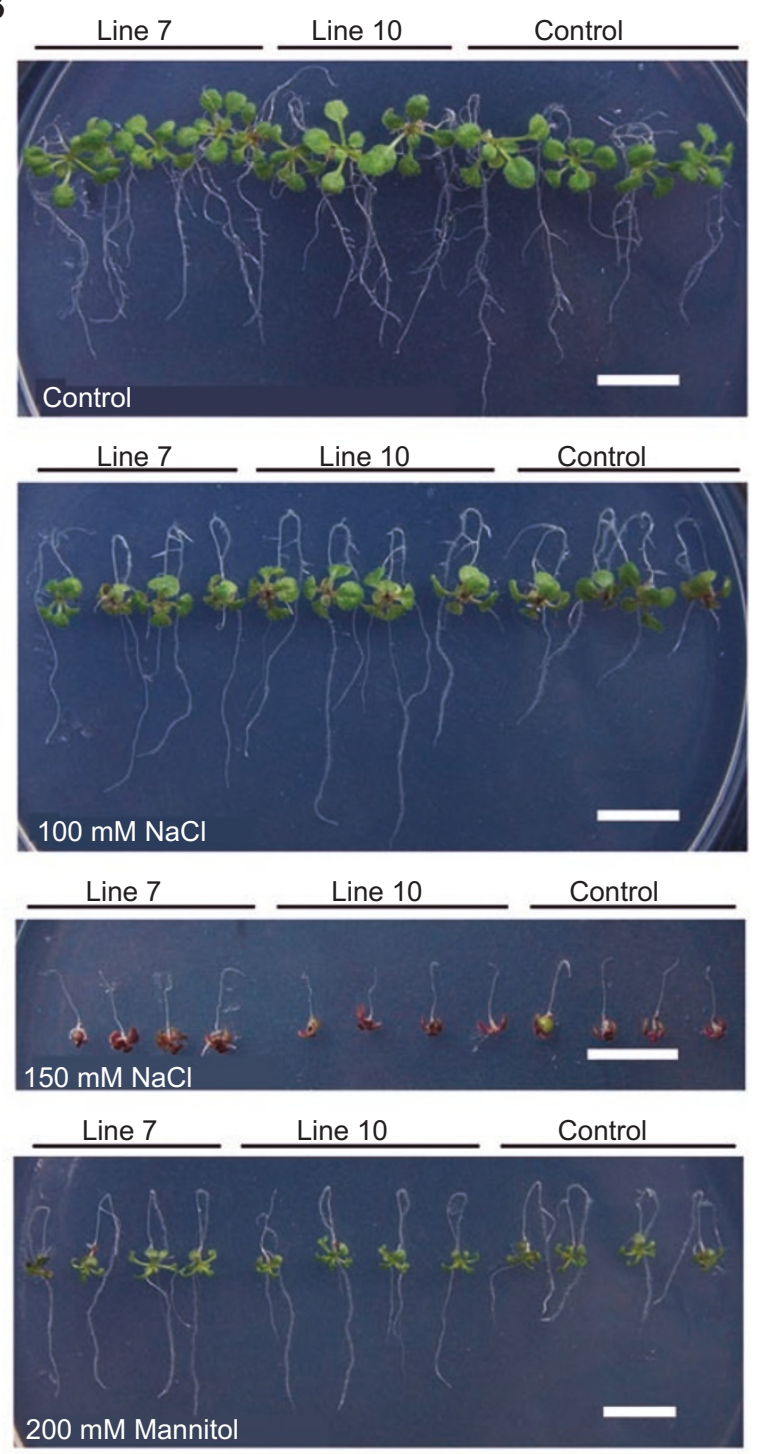

Figure 6 Analysis on the transgenic lines over expressing OsPIP genes. (A) Over-expression of OsPIP1-1 and OsPIP2-2 in transgenic Arabidopsis confirmed by RT-PCR. Up panel represents the expression of OsPIP1-1 in transgenic Arabidopsis line 7 and line 10; low panel represents the expression of OSPIP2-2 in transgenic Arabidopsis line 8 and line 19. Control: the transgenic plants with empty pQGIE110 vector. (B) Root-bending assay for examining the tolerance of salt and drought in transgenic plants over-expressing OsPIP1-1. The first panel: transgenic seedlings on $\mathrm{MS}$ medium without $\mathrm{NaCl}$ or mannitol; the second panel: transgenic seedlings on MS medium with $100 \mathrm{mM} \mathrm{NaCl}$; the third panel: transgenic seedlings on MS medium with $150 \mathrm{mM} \mathrm{NaCl}$; the fourth panel: transgenic seedlings on MS medium with $200 \mathrm{mM}$ mannitol. Control: transgenic plants with empty pQGIE110 vector. Bars: $1 \mathrm{~cm}$. (C) Statistic analysis of root length of transgenic plants over-expressing OsPIP1-1 under MS medium, MS media supplemented with $100 \mathrm{mM}$ of $\mathrm{NaCl}, 150 \mathrm{mM}$ of NaCl , and $200 \mathrm{mM}$ of mannitol for $7 \mathrm{~d}$. Error bars: standard deviation. (D) Statistic analysis of root growth of transgenic plants over-expressing OsPIP2-2 under fresh MS medium, MS media supplemented with $100 \mathrm{mM}$ of NaCl, $150 \mathrm{mM}$ of $\mathrm{NaCl}$ and $200 \mathrm{mM}$ of mannitol for $7 \mathrm{~d}$. Error bars: standard deviation.

Figure 4 shows the expression patterns of $O s P I P$ genes in rice seedlings in response to $250 \mathrm{mM}$ of $\mathrm{NaCl}$. In leaves, the expression levels of most $O s P I P$ s were first suppressed to a certain extent and then increased. The transcript level peaked at $6 \mathrm{~h}$ of the treatment (Figure $4 \mathrm{~A})$. In roots, the expression of all the OSPIP2 genes were not altered or rather slightly suppressed during $24 \mathrm{~h}$ treatment (Figure 4B). It is worth noting that of all the OSPIPI genes increased at 
certain time point of the treatment. For instance, OSPIP1-3 was induced by 2.5 -fold at $2 \mathrm{~h}$ of the treatment and then gradually declined; whereas the expression of OSPIP1-1 increased by about two-fold constantly (Figure 4B).

Treatment with PEG6000 was adopted to mimic the drought stress $[16,32]$. In leaves, OsPIP2-3 was the only gene whose expression was induced by the treatment at 6 $\mathrm{h}$. The expression of other genes such as OSPIP1-2 and OsPIP2-4 were not affected by the treatment, whereas the transcription level of three genes, i.e. OsPIP2-1, OsPIP2-5, and OsPIP2-6, were suppressed (Figure 5A). However, in roots, almost all the OsPIP genes, especially OsPIP1-1, OsPIP2-5 and OSPIP2-7, were found induced during the treatment (Figure 5B), suggesting that $O s P I P$ genes may play important roles in the response to drought in roots.

Over-expression of either OsPIP1 or OsPIP2 could enhance the tolerance of transgenic Arabidopsis to salt and drought treatments

To further characterize the possible function of $O S P I P$ genes, we transformed an OsPIP1 gene (OsPIP1-1) and an OsPIP2 gene (OsPIP2-2) driven by a $35 \mathrm{~S}$ promoter into Arabidopsis thaliana ecotype Columbia respectively and obtained transgenic lines. The expression of the transgenes were confirmed by RT-PCR (Figure 6A). Two over-expression lines for each transgenes, together with the line transformed with an empty vector as control, were examined for their tolerance to salt and drought treatments. When the $O S P I P 1-1$ over-expression lines were treated with 100 $\mathrm{mM}$ of $\mathrm{NaCl}$, a salinity where the growth of the main roots in the control seedlings was inhibited, the lengths of the main roots in two transgenic lines (line 7 and line 10) were not significantly different from those of the plants grown on medium without salt (Figure 6B and 6C). However, when the concentration of $\mathrm{NaCl}$ was increased to $150 \mathrm{mM}$, the growth of both the transgenic and control lines were strongly inhibited (Figure 6B and 6C). Furthermore, when the transgenic lines were exposed to $200 \mathrm{mM}$ of mannitol which was used to mimic drought stress in Arabidopsis [17], the growth of the main roots was not affected compared to the control line (Figure 6B and 6C). Interestingly, similar phenotypes had been observed for the OSPIP2-2 over-expression transgenic lines (Figure 6D).

\section{Discussion}

Similar to the Arabidopsis AtPIP gene family [17], the rice $O S P I P$ familly exhibits significant differences in mRNA abundance among its members (Figure 2). The overall transcript level of OsPIP2-4 was the highest, accounting for about $75 \%$ of the total OsPIP transcripts in the seedlings at two weeks after germination. In roots,
OsPIP2-4 even accounted for $80 \%$ of the total OSPIP transcripts. Therefore, OsPIP2-4, like Arabidopsis AtPIP1-1 and AtPIP 1-2 [17], could be the predominant gene playing critical roles regulating water uptake and transport in rice seedlings, especially in roots. In leaves, OSPIP1-2 accounted for about $35 \%$ of total OSPIP transcripts, which is more than that of OsPIP2-4 (25\%), suggesting an essential role of OSPIP1-2 in water transport in rice leaves. No organ-specific expression pattern for AtPIP genes was reported in Arabidopsis [2, 17]. In this study, we found one leaf-specific OsPIP gene, OsPIP2-6, and three rootspecific genes, OsPIP1-3, OsPIP2-2, and OsPIP2-7 in rice seedlings (Figure 2). ZmPIP1-5 and ZmPIP2-5, the maize homologues of OSPIP1-3 and OSPIP2-2 respectively (Figure 1B), were also detected only in roots $[26,37]$. The organ-specific expression pattern of these OsPIP genes suggested that they could be involved in water uptake and transport in a particular area, like ZmTIP1 in maize [7] and BobTIP26-1 and BobTIP26-2 in cauliflower [27].

The expression of other six OSPIP genes could be detected in both leaves and roots. However, the expression levels of OsPIP1-1, OsPIP1-2 and OSPIP2-1 were higher in leaves than in roots, whereas those of OSPIP2-4 and OSPIP2-5 were higher in roots than in leaves (Figure 2). Similar expression pattern was also reported in other plants. For instance, ZmPIP1-5b, a PIP gene in maize, was found abundant in roots but hardly detected in young and mature leaves [28]. This data may suggest that water homeostasis maintenance in rice leaves and roots may need the coordination of certain OsPIP proteins, or share a relatively redundant system.

Aquaporins play a significant role in recovery from water deficit in both prokaryotic and eukaryotic cells $[1,38]$. Expression of aquaporin genes are regulated under water stress treatments in many plant species [17, 18, 20-22]. In order to understand the nature of aquaporin genes in rice, we analyzed the expression patterns of the ten OSPIP genes under the treatments of ABA, salinity and drought. The OSPIP genes were found showing different responses to different stress treatments. $\mathrm{ABA}$ and $\mathrm{NaCl}$ induced the expression of OSPIPs both in leaves and roots, whereas PEG6000-simulated drought stress mostly induced the expression of these genes in roots (Figure 3-5). Under the same stress, some OsPIP genes showed different inductivity in different organs. For instance, OSPIP2-5 was induced in leaves but not in roots when treated with ABA (Figure 3), whereas OSPIP1-1 was induced in roots but not in leaves when treated with PEG6000 (Figure 5). The different expression pattern may reflect the need for water uptake and transport in different tissues in response to different stresses. Meanwhile, the similar pattern shared by ABA and $\mathrm{NaCl}$ treatments suggests that some OsPIPs might be 
coordinately orchestrated or redundant in the responses to these two stresses.

In Arabidopsis, all the AtPIP genes are either induced or suppressed both in roots and in aerial parts under ABA, salt, cold and drought stimuli. That is to say, the expression pattern of AtPIP genes in roots and the aerial parts of Arabidopsis are always similar in response to the same stress [17]. In rice, however, OsPIP genes expressed in a different, mostly opposite pattern in these two organs. For example, almost all the OsPIPs, especially OSPIP1-1, OsPIP2-5 and OsPIP2-7, were induced in roots by PEG6000 treatment. However, in leaves, only OSPIP2-3 was induced by this treatment, and the expression of other gene members was either not affected or even reduced (Figure 5). This may suggest different roles for OSPIP genes in different organs in response to the same stress. The difference between the expression pattern of PIP genes in the roots of Arabidopsis and rice might be related to their different root systems and habitats, i.e., Arabidopsis plants have taproots and live on land, while rice plants have fibrous roots and usually live in paddy field or wet land. Further studies on these genes are needed in order to reveal their biochemical and/or physiological functions.

The over-expression of either an OSPIP1 or an OsPIP2 gene enhanced the tolerance to the salt and drought treatments in Arabidopsis plants (Figure 6). However, this improvement only occurred when the concentration of $\mathrm{NaCl}$ was below $150 \mathrm{mM}$, or treated with $200 \mathrm{mM}$ of mannitol. Different research groups reached different conclusions on the function of PIP proteins. It has previously been reported that OsPIP1-1 and OsPIP1-3 had ability to transport water in the Xenopus oocytes [30,32], but other studies showed that only PIP2 proteins showed a rapid water transport activity, whereas PIP1s had no or a low water transport activity in the Xenopus oocytes [28, 39]. Our data indicated that the transgenic plants over-expressing $O S P I P 1-1$ or $O_{S-}$ PIP2-2 showed high tolerance to mild salt stress (Figure 6C and 6D), suggesting that $O S P I P$ genes play important roles in regulating water homeostasis, and that both OSPIP1-1 and OSPIP2-2 could possibly have intrinsic water transport ability in planta, which needs to be further confirmed biochemically in vivo. In combination with the Xenopus system and further analyses of transgenic plants over-expressing individual OSPIP gene members, the function of the OsPIPs in water transport should be revealed.

\section{Acknowledgments}

This work was supported by the National Hi-Tech R\&D Program of China (GN 2001AA222261 and 2002AA2Z1001), the State Key Basic Research and Development Plan (Project GN G1999011602 and
G2000016202), the Excellent Young Teachers Program of Ministry of Education of China (to Li Jia Qu). The authors are grateful to Dr Tomohiko Tsuge in Kyoto University, Japan, Dr Fan Chen in the Institute of Genetics and Developmental Biology at the Chinese Academy of Sciences, Beijing, and Dr Hong Wei Xue in the Institute of Plant Physiology and Ecology at the Chinese Academy of Sciences, Shanghai, for their valuable suggestions and comments on the manuscript.

\section{References}

1 Preston GM, Carroll TP, Guggino WB, Arge P. Appearance of water channels in Xenopus oocytes expressing red cell CHIP28 protein. Science 1992; 256:385-387.

2 Johansson U, Karlsson M, Johansson I, et al. The complete set of genes encoding major intrinsic proteins in Arabidopsis provides a framework for a new nomenclature for major intrinsic proteins in plants. Plant Physiol 2001; 126:1358-1369.

3 Johansson I, Karlsson M, Johanson U, et al. The role of aquaporins in cellular and whole plant water balance. BBA-Biomembranes 2000; 1465:324-342.

4 Maurel C. Aquaporins and water permeability of plant membranes. Annu Rev Plant Physiol Plant Mol Biol 1997; 48:399429.

5 Rivers RL, Dean RM, Chandy G, et al. Functional analysis of nodulin 26, an aquaporin in soybean root nodule symbiosomes. J Biol Chem 1997; 272:16256-16261.

6 Barone LM, Shih C, Wasserman BP. Mercury-induced conformational changes and identification of conserved surface loops in plasma membrane aquaporins from higher plants - Topology of PMIP31 from $\beta$-vulgaris L. J Biol Chem 1997; 272:3067230677.

7 Chaumont F, Barrieu F, Herman EM, Chrispeels MJ. Characterization of a maize tonoplast aquaporin expressed in zones of cell division and elongation. Plant Physiol 1998; 117:1143-1152.

8 Chrispeels MJ, Crawford NM, Schroeder JI. Proteins for transport of water and mineral nutrients across the membranes of plant cells. Plant Cell 1999; 11:661-675.

9 Fotiadis D, Jeno P, Mini T, Wirtz S, et al. Structural characterization of two aquaporins isolated from native spinach leaf plasma membranes. J Biol Chem 2001; 276:1707-1714.

10 Agre P. Aquaporin null phenotypes: the importance of classical physiology. Proc Natl Acad Sci U S A 1998; 95:9061-9063.

11 Heymann JB, Engel A. Structural clues in the sequences of the aquaporins. J Mol Biol 2000; 295:1039-1053.

12 Law RJ, Sansom MSP. Water transporters: How so fast yet so selective? Curr Biol 2002; 12:R250-R252.

13 Quigley F, Rosenberg JM, Shachar-Hill Y, Bohnert HJ. From genome to function: the Arabidopsis aquaporins. Genome Biol 2002; 3: RESEARCH0001. Epub 2001 Dec 7.

14 Schaffner AR. Aquaporin function, structure, and expression: are there more surprises to surface in water relations? Planta 1998; 204:131-139.

15 Zardoya R, Villalba S. A phylogenetic framework for the aquaporin family in eukaryotes. J Mol Evol 2001; 52:391-404.

16 Gao YP, Young L, Bonham-Smith P, Gusta LV. Characterization 
and expression of plasma and tonoplast membrane aquaporins in primed seed of Brassica napus during germination under stress conditions. Plant Mol Biol 1999; 40:635-644.

17 Jang JY, Kim DG, Kim YO, Kim JS, Kang HS. An expression analysis of a gene family encoding plasma membrane aquaporins in response to abiotic stresses in Arabidopsis thaliana. Plant Mol Biol 2004; 54:713-725.

18 Kaldenhoff R, Grote K, Zhu JJ, Zimmermann U. Significance of plasmalemma aquaporins for water-transport in Arabidopsis thaliana. Plant J 1998; 14:121-128.

19 Kaldenhoff R, Kolling A, Richter G. Regulation of the Arabidopsis thaliana aquaporin gene AthH2 (PIPIb). J Photochem Photobiol B 1996; 36:351-354.

20 Katsuhara M, Koshio K, Shibasaka M, et al. Over-expression of a barley aquaporin increased the shoot/root Ratio and raised salt sensitivity in transgenic rice plants. Plant Cell Physiol 2003; 44:1378-1383.

21 Liu Q, Umeda M, Uchimiya H. Isolation and expression analysis of two rice genes encoding the major intrinsic protein. Plant Mol Biol 1994; 26:2003-2007.

22 Mariaux JB, Bockel C, Salamini F, Bartels D. Desiccation- and abscisic acid-responsive genes encoding major intrinsic proteins (MIPs) from the resurrection plant Craterostigma plantagineum. Plant Mol Biol 1998; 38:1089-1099.

23 O'Brien M, Bertrand C, Matton DP. Characterization of a fertilization-induced and developmentally regulated plasma-membrane aquaporin expressed in reproductive tissues, in the wild potato Solanum chacoense Bitt. Planta 2002; 215:485-493.

24 Yang L, Zheng BS, Mao CZ, et al. cDNA-AFLP analysis of inducible gene expression in rice seminal root tips under a water deficit. Gene 2003; 314:141-148.

25 Lopez F, Bousser A, Sissoeff I, et al. Characterization in maize of ZmTIP2-3, a root-specific tonoplast intrinsic protein exhibiting aquaporin activity. J Exp Bot 2004; 55:539-541.

26 Chaumont F, Barrieu F, Wojcik E, et al. Aquaporins constitute a large and highly divergent protein family in maize. Plant Physiol 2001; 125:1206-1215.

27 Barrieu F, Thomas D, Marty-Mazars D, et al. Tonoplast intrinsic proteins from cauliflower (Brassica oleracea L. var. botrytis): immunological analysis, cDNA cloning and evidence for expression in meristematic tissues. Planta 1998; 204:335-344.
28 Gaspar M, Bousser A, Sissoeff I, et al. Cloning and characterization of ZmPIP1-5b, an aquaporin transporting water and urea. Plant Sci 2003; 165: 21-31.

29 Grote K, von Trzebiatovski P, Kaldenhoff R. NA levels of plasma membrane aquaporins in Arabidopsis thaliana. Protoplasma 1998; 204:139-144.

30 Li LG, Li SF, Tao Y, Kitagawa Y. Molecular cloning of a novel water channel from rice: its products expression in Xenopus oocytes and involvement in chilling tolerance. Plant Sci 2000; 154:43-51.

31 Malz S, Sauter M. Expression of two PIP genes in rapidly growing internodes of rice is not primarily controlled by meristem activity or cell expansion. Plant Mol Biol 1999; 40:985-995.

32 Lian HL, Yu X, Ye Q, et al. The role of aquaporin RWC3 in drought avoidance in rice. Plant Cell Physiol 2004; 45:481489.

33 Clough SJ, Bent AF. Floral dip: A simplified method for Agrobacterium-mediated transformation of Arabidopsis thaliana 1998; Plant J 16:735-743.

34 Murashige T, Skoog F. A revised medium for rapid growth and bioassays with tobacco tissue cultures. Physiol Plant 1962; 15:473-497.

35 Zhu JK, Liu J, Xiong L. Genetic analysis of salt tolerance in Arabidopsis. Evidence for a critical role of potassium nutrition. Plant Cell 1998; 10:1181-1191.

36 De Groot BL, Grubmuller H. Water permeation across biological membranes: Mechanism and dynamics of aquaporin-1 and GlpF. Science 2001; 294:2353-2357.

37 Murata K, Mitsuoka K, Hirai T, et al. Structural determinants of water permeation through aquaporin-1. Nature 2000; 407:599605.

38 Fetter K, Van Wilder V, Moshelion M, Chaumont F. Interactions between plasma membrane aquaporins modulate their water channel activity. Plant Cell 2004; 16:215-228.

39 Martre P, Morillon R, Barrieu F, et al. Plasma membrane aquaporins play a significant role during recovery from water deficit. Plant Physiol 2002; 130:2101-2110.

40 Siefritz F, Tyree MT, Lovisolo C, et al. PIP1 plasma membrane aquaporins in tobacco: From cellular effects to function in plants. Plant Cell 2002; 14: 869-876.

Edited by Hong Wei Xue 